\title{
Observable consequences of kinetic and thermal AGN feedback in elliptical galaxies and galaxy clusters
}

\author{
Edward C.D. Pope ${ }^{1 \star}$ \\ ${ }^{1}$ School of Physics $\& 3$ Astronomy, University of Victoria, Victoria, BC, V8P 1A1, Canada
}

25 October 2018

\begin{abstract}
We have constructed an analytical model of AGN feedback and studied its implications for elliptical galaxies and galaxy clusters. The results show that momentum injection above a critical value will eject material from low mass elliptical galaxies, and leads to an X-ray luminosity, $L_{\mathrm{X}}$, that is $\propto \sigma^{8-10}$, depending on the AGN fuelling mechanism, where $\sigma$ is the velocity dispersion of the hot gas. This result agrees well with both observations and semi-analytic models. In more massive ellipticals and clusters, AGN outflows quickly become buoyancy-dominated. This necessarily means that heating by a central cluster AGN redistributes the intracluster medium (ICM) such that the mass of hot gas, within the cooling radius, should be $\propto L_{\mathrm{X}}\left(<r_{\text {cool }}\right) /\left[g\left(r_{\text {cool }}\right) \sigma\right]$, where $g\left(r_{\text {cool }}\right)$ is the gravitational acceleration at the cooling radius. This prediction is confirmed using observations of seven clusters. The same mechanism also defines a critical ICM cooling time of $\sim 0.5 \mathrm{Gyr}$, which is in reasonable agreement with recent observations showing that star formation and AGN activity are triggered below a universal cooling time threshold.
\end{abstract}

Key words:

\section{INTRODUCTION}

It is well known that elliptical galaxies are commonly the hosts of powerful AGN (McLure et al. 2004). These sources give rise to lobes of radio emission embedded in the X-ray emitting gaseous haloes surrounding the galaxies and permeating clusters of galaxies (e.g. Burns 1990; Best et al. 2005; Bîrzan et al. 2004; Dunn et al. 2005; Rafferty et al. 2006; Best 2007; Nulsen et al. 2007; Diehl \& Statler 2008).

Recent observational studies of AGN in elliptical galaxies, and galaxy clusters, also suggest that AGN activity is related to the thermal state of its environment. Systems with short radiative cooling times, or a low central entropy, have been shown to be more likely to host active star formation, optical line-emission and jet-producing AGN (e.g. Burns 1990; Crawford et al. 1999; Rafferty et al. 2008; Cavagnolo et al. 2008; Mittal et al. 2008). This suggests that AGN activity is part of a feedback loop that is likely to have important consequences for its environment.

The theoretical evidence for the importance of AGN feedback has also continued to mount. Tabor \& Binnev (1993) and Binney \& Tabor (1995) employed AGN feedback to prevent catastrophic radiative cooling of the $\mathrm{X}$ ray emitting gas that surrounds elliptical galaxies. If un-

* E-mail:ecdpope@uvic.ca opposed, radiative cooling would lead to the deposition of large quantities of cold gas in the central galaxy, and thus cause the galaxy to grow rapidly. Since this appears not to be the case, it was assumed that there must be a heat source that balances the cooling. In a different approach to a similar problem, semi-analytic models of galaxy formation and evolution (e.g. Benson et al. 2003; Croton et al. 2006; Bower et al. 2006) also demonstrated that AGN feedback was necessary to reproduce the observed galaxy luminosity function at large masses, and balance the large radiative cooling rates in clusters of galaxies. In the absence of AGN feedback, the models produced too many massive galaxies.

Semi-analytic models (e.g. Benson et al. 2003), hydrodynamic simulations (e.g. Bregman \& David 1988; Gaetz 1989; Pope et al. 2005) and observations (e.g. Voigt \& Fabian 2004; Pope et al. 2006) have all shown that thermal conduction cannot provide the required energy transfer rates to balance cooling in the majority of cases. However, numerous hydrodynamical simulations have shown that a combination of AGN heating and thermal conduction can maintain the system in a steadystate (e.g. Ruszkowski \& Begelman 2002; Brüggen 2003; Hoeft \& Brüggen 2004; Roychowdhury et al. 2004), see also Guo et al. (2008) for a stability analysis.

Recent theoretical efforts have focussed on trying to understand the X-ray luminosity/temperature $\left(L_{\mathrm{X}}-T\right)$ rela- 
tion in elliptical galaxies and galaxy clusters. The results of semi-analytic models (e.g. Bower et al. 2008; Davé et al. 2008) and hydrodynamic models (e.g. Puchwein et al. 2008) have shown that AGN feedback does indeed play a significant role in governing the scaling of this relation.

The aim of this article is to describe the impact on elliptical galaxies of momentum and thermal energy injection by a central AGN. There are critical thresholds above which each energy component will eject material from the galaxy, and so each mechanism leads to clear observable signatures which can be used to distinguish between them. This study is restricted to the study of galaxies where the X-ray emission is dominated by the gaseous atmosphere, rather than stellar sources (e.g. O'Sullivan et al. 2001).

To this end, we have employed an analytical model of AGN feedback in order to better understand, and compliment, the results that have emerged from semi-analytic and hydrodynamic simulations, as well as observations.

The article is arranged as follows: in section 2 we introduce the basic model describing the effect of energy and momentum injection into a hot gaseous atmosphere. Section 3 contains the results for the limiting cases of momentum dominated and thermally dominated outflows and discusses the implications for the $L_{\mathrm{X}}-\sigma$ relation in elliptical galaxies. Note, we have chosen to study the $L_{\mathrm{X}}-\sigma$ relation rather than $L_{\mathrm{X}}-T$, because the gravitational potential can be written in terms of $\sigma$ which we assume to be unaltered by feedback. In section 4, the theory is applied directly to observations of galaxy clusters. The main findings are summarised in section 5 .

\section{THE MODEL}

The aim of this section is to develop a simple, but useful, analytical formulation that can describe the general impact of AGN feedback on the $L_{\mathrm{X}}-\sigma$ relation of elliptical galaxies. For this purpose the elliptical galaxy is approximated as a singular isothermal sphere (e.g. King 2003, 2005; Murrav et al. 2005). This is because the setup has proved to be a successful starting point in describing phenomena such as the black hole mass/stellar velocity dispersion relation. The results are used to illustrate general behavioural trends, and are not intended to be a complete description of all the physical processes involved. However, in Section 4 , which focuses on galaxy clusters, observational data are used to calculate more accurate gravitational potentials and gas masses. This allows specific predictions of the model to be tested rigorously on individual systems.

For the singular isothermal sphere, the mass of gas within a radius $R$, is

$$
M_{\mathrm{T}}(R)=4 \pi \int_{0}^{R} \rho r^{2} \mathrm{~d} r=\frac{2 f_{\mathrm{g}} \sigma^{2} R}{G},
$$

where $f_{\mathrm{g}} \approx 0.17$ (Spergel et al. 2007) is the gas fraction, $\sigma$ is the velocity dispersion, $R$ is the radius, and $G$ is Newton's gravitational constant. In this model, the AGN resides at the centre of the potential, and produces outflows that propagate radially outwards. The mass swept up by outflows subtending a solid opening angle, $\Omega$, is therefore

$$
M(R)=\left(\frac{\Omega}{4 \pi}\right) \frac{2 f_{\mathrm{g}} \sigma^{2} R}{G},
$$

so that $M(R)=\Omega M_{\mathrm{T}}(R) /(4 \pi)$. As long as the outflow is active, new material from the gaseous atmosphere will be prevented from entering this solid angle through the sides. Therefore, equation(2) gives a reasonable estimate of the mass collected by the outflow. The darkmatter gravitational potential is assumed to be unaffected by the movement of the gas.

Considering momentum injection at a rate of $\dot{P}$, Newton's 2nd law gives

$$
\frac{\mathrm{d}[M(R) \dot{R}]}{\mathrm{d} t}=\dot{P}+g(R) M(R),
$$

where $g(R)$ is the gravitational acceleration. If $\dot{P} \geqslant$ $-g(R) M(R)$, momentum injection will overcome the gravity of the galaxy and the AGN will induce a bulk outflow of hot material collected from the galaxy's atmosphere.

However, the outflow must do work against both gravity and against the pressure of the gaseous atmosphere it is lifting. This means that a term, $(\Omega / 4 \pi) 4 \pi R^{2} p_{\text {amb }}$, must be subtracted from the right hand side of equation (3), where $p_{\text {amb }}$ is the ambient pressure. Using the ideal gas equation, the pressure term can be written

$$
\left(\frac{\Omega}{4 \pi}\right) 4 \pi R^{2} p_{\mathrm{amb}}(R)=\left(\frac{\Omega}{4 \pi}\right) 4 \pi R^{2} \frac{\rho(R) k_{\mathrm{b}} T}{\mu m_{\mathrm{p}}}=\left(\frac{\Omega}{4 \pi}\right) \frac{2 f_{\mathrm{g}} \sigma^{4}}{G},
$$

where $k_{\mathrm{b}} T / \mu m_{\mathrm{p}} \equiv \sigma^{2}$.

The AGN also injects thermal energy, at a rate $\dot{E}_{\text {thermal }}$, that will exert an outward pressure on the ambient gas. Assuming that the inflation is reversible

$$
\dot{E}_{\text {thermal }}=\frac{\gamma}{\gamma-1}\left(\frac{\Omega}{4 \pi}\right) 4 \pi R^{2} \dot{R} p_{\mathrm{amb}}(R),
$$

where $\gamma$ is the adiabatic index of the injected gas. Therefore, we must add a term

$$
\left(\frac{\Omega}{4 \pi}\right) 4 \pi R^{2} p_{\mathrm{amb}}(R)=\frac{\gamma-1}{\gamma} \frac{\dot{E}_{\text {thermal }}}{\dot{R}},
$$

to the right hand side of equation (3). (This term should be compared with the $L_{\text {Eddington }} / c$ term used in King 2003, 2005, to model the outflow momentum.) The completed equation of motion can be written

$$
\frac{\mathrm{d}[M(R) \dot{R}]}{\mathrm{d} t}=\dot{P}+g(R) M(R)-\left(\frac{\Omega}{4 \pi}\right) \frac{2 f_{\mathrm{g}} \sigma^{4}}{G}+\frac{\gamma-1}{\gamma} \frac{\dot{E}_{\text {thermal }}}{\dot{R}} .
$$

\section{RESULTS}

In this section we investigate the behaviour of equation (7) in two limits: momentum-dominated AGN heating, and thermally-dominated AGN heating.

\subsection{Kinetic feedback}

In the limit that the outflow is completely momentum driven, the critical value of the momentum injection rate for the singular isothermal sphere is

$$
\dot{P}_{\text {crit }}=-g(R) M(R)+\left(\frac{\Omega}{4 \pi}\right) \frac{2 f_{\mathrm{g}} \sigma^{4}}{G}=\left(\frac{\Omega}{4 \pi}\right) \frac{6 f_{\mathrm{g}} \sigma^{4}}{G} .
$$


In general, it is more intuitive to consider the energy injection rate rather than the momentum flux. Therefore, equation (8) shall be re-written accordingly. The momentum injection rate is related to the kinetic energy injection rate by

$$
\dot{E}_{\text {kinetic }}=\frac{\dot{P}^{2}}{2 \dot{m}_{\text {out }}}
$$

where $\dot{m}_{\text {out }}$ is the mass flow rate of the jet.

Using equation (9) with equation (8) and rearranging for the critical luminosity gives

$$
\dot{E}_{\text {kinetic,crit }}=\frac{9 \Omega^{2} \sigma^{8} f_{\mathrm{g}}^{2}}{8 \pi^{2} G^{2} \dot{m}_{\text {out }}}
$$

which, in scaled units, is

$$
\begin{array}{r}
\dot{E}_{\text {kinetic,crit }} \sim 2 \times 10^{42}\left(\frac{\Omega / 4 \pi}{0.02}\right)^{2}\left(\frac{\dot{m}_{\text {out }}}{10 \mathrm{M}_{\odot} \mathrm{yr}^{-1}}\right)^{-1} \\
\left(\frac{\sigma}{200 \mathrm{~km} \mathrm{~s}^{-1}}\right)^{8} \mathrm{erg} \mathrm{s}^{-1} .
\end{array}
$$

The reason $\Omega / 4 \pi=0.02$ has been chosen will become apparent in section 4 , where the value is derived from observations. At present, it is sufficient to note that $\Omega$ is an effective opening angle, rather than the actual opening angle of the outflow. It is the solid angle within which the outflow interacts with ambient material, and is therefore larger than the typical value dervied from radio observations (e.g. see Binney \& Tabor 1995, and references therein).

The luminosities hinted at in equation (11) mean that it is plausible to expect kinetic AGN feedback to eject hot material from the atmosphere of the galaxy. This will have two effects: it will reduce the X-ray luminosity of the galaxy, $L_{\mathrm{X}}$, and reduce the gas fraction.

In clusters (with large $\sigma$ ), the critical jet power will exceed the cluster X-ray luminosity, and would require implausibly large jet powers to eject mass. This means that the AGN jets are unlikely to significantly affect the $L_{\mathrm{X}} \propto \sigma^{4.4}$ relation (Mahdavi \& Geller 2001) in clusters. Of course, this does not mean that they do not play a significant role in heating the inner regions of such objects.

To make a realistic estimate of the $L_{\mathrm{X}}-\sigma$ relation expected as a consequence of momentum-dominated feedback, we follow the method of Bower et al. (2008). Their prescription for the effect of the AGN activity was to remove mass from the galaxy at a rate governed by the difference between the heating and cooling rates: $\dot{m}=2\left(\dot{E}-L_{\mathrm{X}}\right) / \sigma^{2}$. Our model is expected to behave in a similar way, but with the reference point being the critical jet power defined in equation (11)

$$
\dot{m}=\frac{2\left(\dot{E}_{\text {kinetic }}-\dot{E}_{\text {crit }}\right)}{\sigma^{2}} .
$$

Equation (12) tells us that the mass-loss from the galaxy will effectively stop when the average feedback heating rate becomes comparable with the critical jet power for that gravitational potential.

The complete derivation requries a functional form for

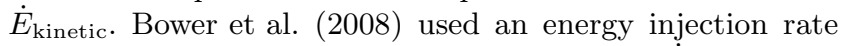
that is proportional to the mass cooling rate, $\dot{M}_{\text {cool }}$, of the $\mathrm{X}$-ray emitting atmosphere around the galaxy. We will follow their example as an illustrative case. Therefore

$$
\dot{E}_{\text {kinetic }}=\eta \delta \dot{M}_{\mathrm{cool}} c^{2},
$$

where the factor $\delta$ accounts for the fraction of cooling material that reaches the black hole. Here, $\delta$ is also used to account for the reduced cooling rate compared to the classical value. Given that cooling in such systems appears to be reduced by a factor of 10 , and assuming that only one tenth of the inflowing material reaches the black hole, $\delta \sim 0.01 . \eta$ is the accretion efficiency, and $c^{2}$ is the speed of light. The classical mass cooling rate can be written in terms of the X-ray luminosity of the hot gas, as

$$
\dot{M}_{\text {cool }}=\frac{2}{5} \frac{L_{\mathrm{X}}}{\sigma^{2}}
$$

so that the kinetic energy injection rate is

$$
\dot{E}_{\text {kinetic }}=0.4 \eta \delta L_{\mathrm{X}} \frac{c^{2}}{\sigma^{2}} .
$$

Substituting equation (15) into equation (12) shows that the mass-loss rate from the galaxy will tend to zero as

$$
L_{\mathrm{X}} \sim 2.5 \frac{\sigma^{2} \dot{E}_{\text {kinetic,crit }}}{\eta \delta c^{2}} \propto \sigma^{10}
$$

In scaled units, this means we expect the X-ray luminosity of an elliptical galaxy to be approximately

$$
\begin{array}{r}
L_{\mathrm{X}} \sim 2 \times 10^{39}\left(\frac{\Omega / 4 \pi}{0.02}\right)^{2}\left(\frac{\delta}{0.01}\right)^{-1}\left(\frac{\dot{m}_{\text {out }}}{10 \mathrm{M}_{\odot \mathrm{yr}^{-1}}}\right)^{-1} \\
\left(\frac{\sigma}{200 \mathrm{~km} \mathrm{~s}^{-1}}\right)^{10} \operatorname{erg~s}^{-1}
\end{array}
$$

where an accretion efficiency of $\eta=0.1$ has been assumed. These values agree reasonably well with the observed X-ray luminosities of elliptical galaxies (e.g. Fukazawa et al. 2006). The result agrees well with the observations presented by Mahdavi \& Geller (2001) who also found $L_{\mathrm{X}} \propto \sigma^{10}$, and is consistent with the relation found by O'Sullivan et al. (2003). The normalisation of the relation found by Mahdavi \& Geller (2001) seems to be slightly higher than equation (17), possibly suggesting that $\delta \sim 10^{-3}$ would be a better description of the physics governing the accretion rate. This would guarantee a low Eddington fraction accretion rate which would produce outflows (e.g. Maccarone et al. 2003) and not quasars.

The scaling relation in equation (17) is steeper than the results of numerical simulations performed by Davé et al. (2008) who found $L_{\mathrm{X}} \propto \sigma^{8.2}$. However, our model would also produce $L_{\mathrm{X}} \propto \sigma^{8}$ if the AGN fuelling rate was proportional to $L_{\mathrm{X}}$ rather than $L_{\mathrm{X}} / \sigma^{2}$. In both observations and simulations, there is significant scatter in these relationship. Presumeably this could be caused by the different histories of individual galaxies (mergers and ages) and also the stochastic nature of AGN feedback.

\subsection{Thermal energy injection}

It is also important to consider the role of thermal energy injection. According to equation (7), the effect of thermal injection should be quite different to pure momentum injection. This leads to several predictions which can be tested against existing observations.

A variant of this mechanism is generally considered to lead to the black hole mass - bulge stellar velocity dispersion relation (e.g. Puchwein et al. 2008). However, in their approach the radiative power output of the AGN accretion 
at a high Eddington ratio couples to the ambient gas. We will not be considering effects of radiation output from the AGN since it is assumed here that the AGN simply injects thermal energy that does work against its surroundings, rather than assuming anything more complex.

Using equation (7), the critical energy injection rate for the thermal gas to eject material from the gravitational potential is of the order

$$
\dot{E}_{\text {thermal,crit }} \sim\left(\frac{\Omega}{4 \pi}\right) \frac{6 f_{\mathrm{g}} \sigma^{5}}{G},
$$

where we have set $\dot{R} \sim \sigma$. In scaled units this gives

$$
\dot{E}_{\text {thermal,crit }} \sim 10^{42}\left(\frac{\Omega / 4 \pi}{0.02}\right)\left(\frac{\sigma}{200 \mathrm{~km} \mathrm{~s}^{-1}}\right)^{5} \mathrm{erg} \mathrm{s}^{-1} .
$$

In reality, the AGN injects both momentum and thermal energy (as well as the radiation that we are not considering), rather than one or the other. The behaviour of the system will therefore depend on the relative proportions.

For simplicity, the thermal energy injection rate can be written as a fraction, $\chi$, of the total energy injection rate; $\dot{E}_{\text {thermal }}=\chi \dot{E}_{\text {total }}$. Therefore, the critical threshold for thermal energy injection, in terms of the total energy injection rate, is $\dot{E}_{\text {total,crit }}=\dot{E}_{\text {thermal,crit }} / \chi$. It is probably safe to assume that initially, at least, outflows are momentum dominated, since they are highly anisotropic. This suggests that $\chi \ll 1$. In the next section we will show that, above a critical jet length, AGN outflows will become buoyancy dominated so that $\chi \rightarrow 1$ for large radii. However, for the remainder of this section it is assumed that the relative fractions of kinetic and thermal energy in the outflow remain constant, and that the outflows are momentum dominated.

Combining the critical luminosities for both thermal and kinetic limits we find that the critical energy injection rate can be expressed as a broken power-law that scales as $\sigma^{8}$ at lower masses, and $\sigma^{5}$ at higher masses. The location of the break depends on the value of $\chi ;\left(\sigma_{\text {break }} / 200 \mathrm{~km} \mathrm{~s}^{-1}\right) \sim$ $(2 \chi)^{-1 / 3}$. So, as an example, if $\chi=0.1$, the break will occur at $\sigma \sim 340 \mathrm{~km} \mathrm{~s}^{-1}$. Above this value of $\sigma$, the criterion for mass ejection from the galaxy follows the thermal limit, while below this the mass ejection criterion is governed by momentum injection. Another way of expressing this is that thermal energy injection has much more important consequences for the galaxy properties at high masses, while momentum injection is more important at lower masses. This result is shown in figure 1.

The critical energy injection rate at $\sigma \sim 340 \mathrm{~km} \mathrm{~s}^{-1}$ is $\sim 10^{44} \mathrm{erg} \mathrm{s}^{-1}$ which is well below the Eddington limit for a $10^{8} M_{\odot}$ black hole. This strongly suggests that thermal feedback from black holes could have an effect on the $L_{\mathrm{X}}-\sigma$ relation in massive elliptical galaxies and groups. However, for a cluster gravitational potential, $\sigma>500 \mathrm{~km} \mathrm{~s}^{-1}$. The critical energy injection rate at $\sigma=500 \mathrm{~km} \mathrm{~s}^{-1}$, is $\sim 10^{47} \mathrm{erg} \mathrm{s}^{-1}$, which is comparable to the Eddington limit for a $10^{9} M_{\odot}$ black hole. Furthermore, depending on the value of $\delta$ the actual power available from accretion might be much lower than this. Consequently, there should be a second break in the $L_{\mathrm{X}}-\sigma$ relation at $\sigma \sim 500 \mathrm{~km} \mathrm{~s}^{-1}$. Assuming that $\sigma^{2}=k_{\mathrm{b}} T / \mu m_{\mathrm{p}}, \sigma=500 \mathrm{~km} \mathrm{~s}^{-1}$ corresponds to a temperature of $\sim 1-2 \mathrm{keV}$, which is approximately where a break is observed (e.g. Xue \& Wu 2000). This, therefore, marks the transition above which black holes are unlikely to affect the

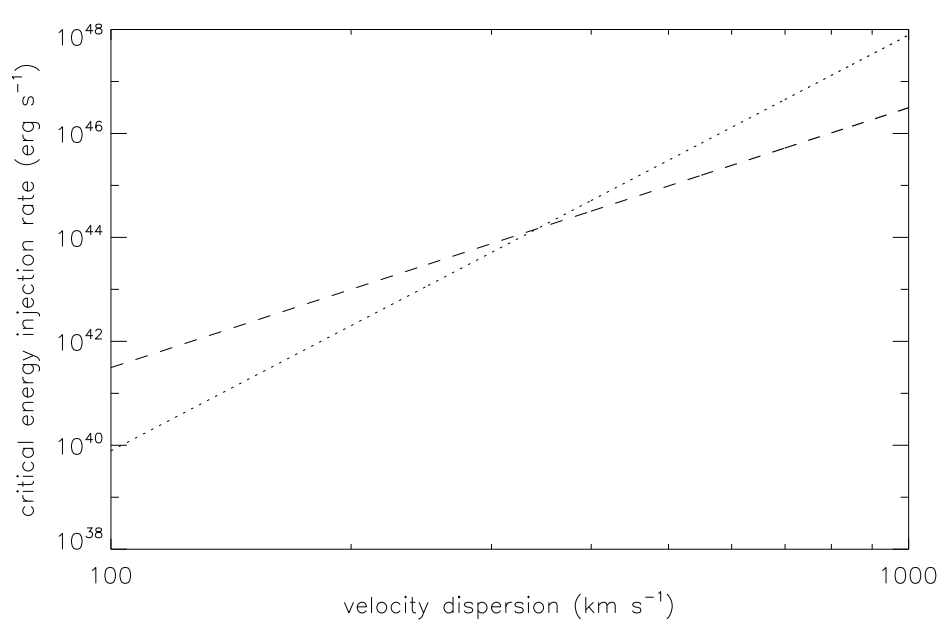

Figure 1. The critical energy injection rate for thermal (dashed line) and momentum (dotted line) injection. In this example it was assumed that the thermal fraction, $\chi=0.1$ so that the critical values cross at roughly $340 \mathrm{~km} \mathrm{~s}^{-1}$. At low $\sigma$, the lower critical energy injection limit is provided by the momentum criterion, while at high $\sigma$ the lower critical injection limit is provided by thermal energy criterion.

global $L_{\mathrm{X}}-\sigma$ relation. Nevertheless, black holes may still be effective in heating the central regions of clusters.

If the accretion rate onto the black hole is $\propto L_{\mathrm{X}} / \sigma^{2}$, as suggested in the previous example, thermal AGN feedback should lead to $L_{\mathrm{X}} \propto \sigma^{7}$ in higher mass ellipticals and groups. Alternatively, if the black hole accretion rate is $\propto L_{\mathrm{X}}$, the model would lead to $L_{\mathrm{X}} \propto \sigma^{5}$.

So, in summary, the model predicts $L_{\mathrm{X}} \propto \sigma^{8-10}$ for lower masses elliptical galaxies, $L_{\mathrm{X}} \propto \sigma^{5-7}$ for higher mass ellipticals and groups, depending on the AGN fuelling mechanism. However, given that the relation for clusters can be written $L_{\mathrm{X}} \propto \sigma^{4.4}$ Mahdavi \& Geller 2001) and there is a noticeable break in the scaling at $\sim 1-2 \mathrm{keV}$ (e.g. Xue \& Wu 2000) (or $\sigma \sim 500 \mathrm{~km} \mathrm{~s}^{-1}$ ), it seems that an AGN fuelling rate that is $\propto L_{\mathrm{X}} / \sigma^{2}$ can best explain the observed $L_{\mathrm{X}}-\sigma$ relation across the mass range considered here.

\section{APPLICATION TO GALAXY CLUSTERS}

Having considered the implications of momentum and thermal energy injection on elliptical galaxies, the model is applied to clusters of galaxies. Because of the stronger gravity, these systems behave rather differently to elliptical galaxies. This leads to several observable consequences which are discussed below. In addition, the benefit of using the singular isothermal sphere for elliptical galaxies was to construct simple scaling laws across the population of galaxies that result from different properties of AGN feedback. However, the previous section demonstrated that AGN feedback is unable to alter the global $L_{\mathrm{X}}-\sigma$ relation in clusters, so using the same general approach would not yield interesting results for clusters. Instead, it seems more appropriate to focus on the central regions of clusters, where the AGN is likely to have an impact and so attempt to discern any observable 
signatures of AGN feedback. To do this accurately requires observations of the temperature and density profiles of the central ICM in a sample of clusters. This is done in Section 4.2. Firstly, though, there is brief discussion of the general effect of the cluster potential on AGN outflows.

\subsection{Buoyancy, work and bubbles in the intracluster medium}

Momentum and thermal energy fractions govern various properties of the outflow, including its morphology. These fractions are not constant, but vary as the outflow propagates through its surroundings. For example, thermal energy injection produces buoyant material, and the buoyancy force is also a function of the local gravitational acceleration. This suggests that identical jets will appear morphologically different in different environments. In particular, buoyancy is more important in more massive ellipticals, and in clusters, due to the stronger gravity in such enviroments. It, therefore, stands to reason that we might expect outflows to appear more bubble-like (buoyant), and less elongated, in clusters. This possibility is investigated below.

For a given thermal energy injection rate, it is possible to calculate a buoyancy flux. An outflow of velocity, $w$, and width, $D$, has a buoyancy flux defined as (e.g. Carlotti \& Hunt 2005, and references therein)

$$
B \equiv \frac{\Delta \rho}{\rho} g w \frac{\pi D^{2}}{4} \mathrm{~cm}^{4} \mathrm{~s}^{-3},
$$

where $g$ is the gravitational acceleration, $\rho$ is the ambient density, and $\Delta \rho$ is the density difference between the outflow and the ambient material. The buoyancy flux can also be written in terms the heating rate that caused the density change (e.g. Carlotti \& Hunt 2005)

$$
B=\frac{\gamma-1}{\gamma} \frac{g \dot{E}_{\text {thermal }}}{p_{\text {amb }}} \mathrm{cm}^{4} \mathrm{~s}^{-3}
$$

where $p_{\text {amb }}$ is the pressure of the ambient gas. Similarly, the momentum flux is defined as (e.g. Carlotti \& Hunt 2005)

$$
U \equiv\left(\frac{\rho_{\text {outflow }}}{\rho}\right) w^{2} \frac{\pi D^{2}}{4}=\frac{\dot{P}}{\rho} \mathrm{cm}^{4} \mathrm{~s}^{-2},
$$

where $\rho_{\text {outflow }} / \rho$ is the ratio of the density in the jet to the ambient density.

It is useful to construct a characteristic length-scale from the momentum and buoyancy fluxes. This length-scale marks the transition from a momentum-dominated flow to a buoyancy-dominated flow and is defined as

$$
l \equiv \frac{U^{3 / 4}}{B^{1 / 2}}
$$

Using the fact that $\dot{P}=\left(2 \dot{m}_{\text {out }} \dot{E}_{\text {kinetic }}\right)^{1 / 2}$, the length-scale can be written

$$
\begin{array}{r}
l \sim 24 \operatorname{kpc}\left(\frac{\dot{m}_{\text {out }}}{10 \mathrm{M}_{\odot} \mathrm{yr}^{-1}}\right)^{3 / 8}\left(\frac{\dot{E}_{\text {kinetic }}}{10^{42} \mathrm{erg} \mathrm{s}^{-1}}\right)^{3 / 8} \\
\left(\frac{\rho}{10^{-25} \mathrm{~g} \mathrm{~cm}^{-3}}\right)^{-3 / 4}\left(\frac{p_{\text {amb }}}{10^{-10} \mathrm{erg} \mathrm{cm}^{-3}}\right)^{1 / 2}\left(\frac{\dot{E}_{\text {thermal }}}{10^{42} \mathrm{erg} \mathrm{s}^{-1}}\right)^{-1 / 2} \\
\\
\left(\frac{g}{10^{-8} \mathrm{cms}^{-2}}\right)^{-1 / 2} .
\end{array}
$$

We have assumed a uniform density for the environment, and constant gravity, but nevertheless, the critical length above which the jet dynamics become dominated by buoyancy is likely to be $\sim 20 \mathrm{kpc}$.

The parameters that control this length-scale show that an outflow will become buoyancy-dominated closer to its origin in a denser environment with stronger gravity. This confirms the earlier statement that, for a given energy injection rate, bubble-like outflows will be more prevalent in the centres of clusters of galaxies than in low mass elliptical galaxies. It also means that more of the injected energy is trapped within the central regions, providing more efficient heating in a cluster environment.

In clusters, it is not possible to relate many of the properties, such as density and pressure, to $\sigma$. As a result, it is not particularly useful to represent equation (24) in terms of the velocity dispersion. In addition, there is no single value of $\sigma$ when the jets become buoyant, because this will depend on the kinetic and thermal energy injection rates and the other cluster properties.

Another possible explanation for the presence of AGNblown bubble-like structures in clusters is that jets never exceed the critical energy injection to eject mass from a cluster. Therefore, the jet will always be constrained by the ambient medium. In contrast, it is possible for the AGN to exceed the critical luminosity in an elliptical galaxy, in which case the jet is no longer constrained. It is possible that this effect plays some role in the apparent FRI/FRII dichotomy. For example, Kaiser \& Best (2007) suggested that FRIs were those objects which are disrupted within the core region the surrounding atmosphere, while FRIIs were jets which were sufficiently powerful to penetrate into the region outside the core where the ambient pressure drops off rapidly. Note, however, other effects such as black hole spin are also a possible explanation for the dichotomy (e.g. Meier 1999).

It is also necessary to show that the term $\dot{E}_{\text {thermal }} / \dot{R}$ term in equation (7) is equivalent to the work done by bouyancy against the surrounding gas. The rate of change of the buoyancy force, according to Archimedes' principle, can be written

$$
\dot{F}=\left(\frac{\Omega}{4 \pi}\right) 4 \pi \rho g R^{2} \dot{R},
$$

where the density of the outflow is assumed to be much less than the material it has displaced. Using equation (21) the rate of change buoyancy force in terms of the heating rate, $\dot{E}_{\text {thermal }}$, is

$$
\dot{F}=\frac{\gamma-1}{\gamma} \frac{\mu m_{\mathrm{p}} g \dot{E}_{\text {thermal }}}{k_{\mathrm{b}} T} .
$$

Equations (25) and (26) can then be rearranged to show that the two forms are equivalent

$$
\frac{\gamma-1}{\gamma} \frac{\dot{E}_{\text {thermal }}}{\dot{R}}=\left(\frac{\Omega}{4 \pi}\right) 4 \pi p_{\mathrm{amb}} R^{2} .
$$

This means that buoyancy has the same effect as thermal energy injection doing work against its surroundings. Furthermore, since the outflows become buoyancy-dominated on length-scales greater than $l$, this means that the initial (24)omentum flux is converted into thermal energy. The total power must be conserved, so for $R>l$, the outflow can be adequately described by $\dot{E}_{\text {thermal }} \sim \dot{E}_{\text {total }}$, where $\dot{E}_{\text {total }}$ is 
the (constant) total energy injection rate of the outflow. As a result, the factor $\chi$, introduced earlier, is a strong function of radius and becomes close to unity outside $R=l$.

Equation (24) suggests that $l$ is likely to be significantly less than the cooling radius in a typical cluster $(\sim 100 \mathrm{kpc})$. Therefore, we argue that on spatial scales comparable with the cooling radius, it is sufficient to model heating by an AGN at the cluster centre, purely in terms of the injection of thermal energy within a given solid angle. To understand the dynamical structures on smaller scales we must consider the jet momentum in detail, but on intermediate spatial scales it seems that the energy injection can be treated as purely thermal. We will next consider the physical implications of thermal heating in galaxy clusters.

\subsection{Observational consequences of buoyancy in cluster cores}

In clusters, the AGN cannot eject gas from the cluster due to the sheer weight of material residing in the gravitational potential. However, there is still a region near the cluster centre where the AGN can affect the distribution of the X-ray emitting material. Since we are considering clusters, buoyancy is important and the energy injection can be thought of as purely thermal. Therefore, the appropriate critical energy injection rate is the thermal injection limit; $\dot{E}_{\text {crit }} \sim-(15 / 4) \sigma M(R) g(R)$. As usual, $M(R)$ is the mass of the material swept up within $R$ and within the solid angle of the outflow; $M(R)=\Omega \int_{0}^{R} r^{2} \rho(r) \mathrm{d} r=\Omega M_{\mathrm{T}}(R) /(4 \pi)$. $\sigma$ is the velocity dispersion of the gravitational potential, and $g(R)$ is the gravitational acceleration at $R$. In general, clusters are close to hydrostatic equilibrium so that the gravitational acceleration can be calculated from the pressure gradient and the gas density; $g(R) \approx \nabla p(R) / \rho(R)$. The multiplicative factor $15 / 4$ comes from $(3 / 2) \times \gamma /(\gamma-1)$, assuming that $\gamma=5 / 3$. Finally, the factor $3 / 2$ is from the contribution of the ambient pressure to the total work required for the thermal outflow to eject material, see equation (7).

For energy injection rates less than this value, material can build up in the central regions of the cluster, while for larger energy injection rates, mass is ejected from the central regions. This is essentially the same as the explanation for mass loss from the elliptical galaxies, except that in clusters mass is ejected from the central regions, but still retained by the cluster as a whole. Of course, this implicitly assumes that some of the energy injected by the AGN is distributed over $4 \pi$ steradians. This could occur through shocks generated in the early stages of outflow and subsequently sound waves (e.g. Voit \& Donahue 2005), or bulk motions in the ICM (e.g. Heinz et al. 2006), though these mechanisms cannot be accounted for in the current model.

The general argument outlined above suggests that there will be a radius, governed by the energy injection rate, within which the weight of X-ray emitting material is governed by AGN feedback. This is important, since by controlling the mass of gas in the central regions of the cluster the AGN also regulates the cooling rate, and in doing so its fuel own supply, and so on.

For clusters, the obvious region of importance is enclosed by the cooling radius, since it contains the material that could cool within the age of the Universe. Therefore, if AGN feedback balances the radiative losses within the cooling radius, $\dot{E}=L_{\mathrm{X}}\left(<r_{\text {cool }}\right)$, then, according to the model, the heating should also redistribute the material in the centre of the cluster. This interplay should have reached a quasi-equilibrium when

$$
\begin{aligned}
\dot{E}=L_{\mathrm{X}} & \left(<r_{\mathrm{cool}}\right)=-\left(\frac{15}{4}\right)\left(\frac{\Omega}{4 \pi}\right) \sigma M\left(r_{\mathrm{cool}}\right) g\left(r_{\mathrm{cool}}\right) \sim \\
& -4 \pi\left(\frac{15}{4}\right)\left(\frac{\Omega}{4 \pi}\right)\left[\frac{\nabla p\left(r_{\mathrm{cool}}\right)}{\rho\left(r_{\mathrm{cool}}\right)}\right] \sigma \int_{0}^{r_{\mathrm{cool}}} r^{2} \rho(r) \mathrm{d} r .
\end{aligned}
$$

In other words, the total mass of X-ray emitting material within $r_{\text {cool }}$, multiplied by the gravitational acceleration at $r_{\text {cool should be }}$

$$
-M_{\mathrm{T}}\left(r_{\mathrm{cool}}\right) g\left(r_{\mathrm{cool}}\right)=\left(\frac{4}{15}\right)\left(\frac{4 \pi}{\Omega}\right) \frac{L_{\mathrm{X}}\left(<r_{\mathrm{cool}}\right)}{\sigma} .
$$

To be strictly correct, the AGN removes a fraction, $\Omega / 4 \pi$, of this material, so the remaining weight must be $-(1-$ $\Omega / 4 \pi) M_{\mathrm{T}}\left(r_{\text {cool }}\right) g\left(r_{\text {cool }}\right)$.

For comparison, AGN energy injection rates can be estimated from observations of bubble enthalpies divided by a characteristic timescale (e.g. Bîrzan et al. 2004; Dunn et al. 2005; Rafferty et al. 2006). Although these values are somewhat uncertain (see Nusser et al. 2006; Binney et al. 2007, for example), it seems that more powerful AGN outbursts do seem to be observed in more luminous clusters as well as those systems with shorter central cooling times. Thus, AGN outbursts in clusters are capable of injecting energy at the rate required by the model.

Equation (29) has been verified by examining the sample of seven clusters discussed in Pope et al. (2006) and can be seen in figure 2 . The sample provides a readily available data set with all the physical parameters required to investigate the model predictions. The main reason for this is that the clusters are nearby so that the temperature and the density profiles are fairly well determined to within a few kiloparsecs of the cluster centre. Accurate temperature and density profiles permit reasonable estimates of the total gas mass, and gravitational acceleration, thus increasing the possibility of detecting subtle variations due to AGN feedback. Each member of the sample is known to host a central AGN, thus making an ideal testbed for the model. Due to the paucity of very high X-ray luminosity clusters, future work will focus on extending the sample predominantly at low X-ray luminosities, to investigate and refine the model.

The best-fit in figure 2 implies a typical solid opening angle of $\Omega / 4 \pi \approx 0.028$, which informed the value of the opening angle used throughout the article. Noting that this value is twice the effective solid opening angle of the outflow, the effective half opening angle of a single outflow must be $\sim 14^{\circ}$. This is a factor of $\sim 3$ greater than the typical values found by radio astronomers (e.g. see Binnev \& Tabor 1995, and references therein). Possible explanations for this are discussed below.

In the simplest case, a buoyant bubble inflated by an AGN would expand adiabatically to maintain pressure equilibrium with its surroundings. According to this model, the bubble radius should behave in the following manner (e.g. Diehl et al. 2008)

$$
\frac{r_{\mathrm{b}}(R)}{r_{\mathrm{b}, 0}}=\left[\frac{p_{\mathrm{amb}}(R)}{p_{0}}\right]^{1 / 3 \Gamma},
$$

where $r_{\mathrm{b}}(R)$ is the bubble radius as a function of radial dis- 
placement from its original location. $r_{\mathrm{b}, 0}$ is the initial radius of the bubble, $p_{0}$ is the pressure at this location, and $p_{\mathrm{amb}}(R)$ is the ambient pressure as a function of radius. $\Gamma$ is the polytropic index of material inside the bubble. The half opening angle, $\theta$, can then be calculated from $\tan (\theta)=\mathrm{d} r_{\mathrm{b}}(R) / \mathrm{d} R$. Substituting suitable values for $r_{\mathrm{b}, 0}$, and $\Gamma=4 / 3$ and using a generic cluster pressure profile suggests that the adiabatic expansion of bubbles would not result in large enough opening angles to explain the best-fit in figure 2. In a more realistic model, Pavlovski et al. (2007) and Pavlovski et al. (2008) showed that the Kutta-Zhukovsky force causes bubbles to expand further than would be expected from pressure equilibrium arguments.

Probably the most plausible explanation is that the effective opening angle derived from figure 2, refers to the entrainment of material from the boundary layer between the outflow and the ambient medium. More specifically, entraining material along the length of the outflow, as well as through the head of the outflow. Consequently, the outflow can collect material from within a larger solid angle than is carved out by the main flow itself, (see Bicknell 1994; Laing \& Bridle 2002, for example).

Generally, $\theta$ is related to the entrainment coefficient, $\alpha$, by $\alpha=\tan (\theta)$ (e.g. Turner 1986). In a uniform ambient medium, and in the Boussinesq limit, the typical entrainment coefficient for a momentum driven outflow is $\alpha \sim 0.05$, but $\alpha \sim 0.08$ for a buoyancy driven flow (see Turner 1986, and references therein). The exact values depend slightly on whether the cross-section of the outflow is modelled as a Gaussian or top-hat function, but the general result is that entrainment coefficients are larger for buoyancy driven flows.

The entrainment coefficient also varies with the density contrast between the outflow and the ambient material, and is a function of the Mach number of the flow. De Young (1993) suggests the opening angle can be written as

$$
\tan (\theta) \sim 0.08\left(\frac{\rho}{\rho_{\text {outflow }}}\right)^{1 / 2}\left(\frac{1}{M}\right)
$$

where $\rho$ is the ambient density, $\rho_{\text {outflow }}$ is the density of the outflow, and $M$ is the Mach number of the flow relative to the ambient material.

The best-fit from figure 2 suggests $\tan (\theta)=\alpha \sim 0.25$. Typical values for a momentum dominated outflow might be $M \sim 10$, and $\rho / \rho_{\text {outflow }} \sim 10^{3}$ which would give $\tan (\theta) \sim 0.25$, using equation (31). In the buoyancy dominated regime, the flow speed can be described by $M \sim 1$, for which equation (31) suggests $\rho / \rho_{\text {outflow }} \sim 10$. The numbers used in this calculation are likely to be common to all clusters, suggesting that entrainment of material might well be what sets the effective opening angle. Furthermore, if the outflows can be described as $\rho_{\text {outflow }} w^{2}=$ constant, as expected for momentum conservation, then the effective opening angle will also be constant, according to equation (31).

In figure 2, A478 appears not to be well-described by the best-fit through the data. This may be because the best-fit assumes a uniform effective opening angle for all the outflows in the cluster sample, and the outflows in A478 typically have a larger effective opening angle. There does not seem to be any particular reason to expect this ; altough A478 is the most massive cluster in this sample, it does not exhibit a gravitational acceleration that is significantly different in magnitude to any other clusters in the sample.

As an alternative explanation, it is interesting to note the large temperature decrement in the cluster centre of A478 (Sun et al. 2003), which is often taken to be a signature of a strong inflow. The AGN also appears to be injecting energy at a very low rate compared to the radiative losses (e.g. see Bîrzan et al. 2004). As a result, it might be no coincidence that A478 seems to have a larger mass within $r_{\text {cool }}$ than would be expected from the best-fit to the data.

Table 1 lists the X-ray luminosities, weights and velocity dispersions used to plot figure 2 . For consistency with our earlier calculations it was assumed that $\sigma^{2}=k_{\mathrm{b}} T / \mu m_{\mathrm{p}}$, where the temperature was taken at its highest value within the cooling radius. The values in table 1 have been rounded to two significant figures. For this reason, and the small sample size, the uncertainties were not calculated since they do not provide any extra information.

An alternative explanation for this correlation is not obvious. So, it seems plausible to conclude that energy injection by AGN does reshape the gas profiles in the central regions of clusters. As a consequence, it is not a surprise that heating and cooling rates match relatively well - because the systems were reconfigured into such a state by the AGN.

Further insight into the interaction between the AGN and the ambient material can be gained by studying equation (29) in more detail. If the equilibrium condition is met at all radii, then

$$
L_{\mathrm{X}}(<R)=-\left(\frac{15}{4}\right)\left(\frac{\Omega}{4 \pi}\right) \sigma M(R) g(R) .
$$

The X-ray luminosity is defined by $L_{\mathrm{X}} \approx \int n^{2} \Lambda(T) \mathrm{d} V$, where $n$ is the electron number density of the gas, and $\Lambda(T)$ is the temperature dependent cooling function. For typical cluster temperatures we expect the emission to be dominated by thermal bremsstrahlung so $\Lambda(T) \sim T^{1 / 2}$.

Differentiating both sides of equation (32), with repsect to volume, leaves

$$
n^{2} \Lambda(T)=-\left(\frac{15}{4}\right)\left(\frac{\Omega}{4 \pi}\right) \sigma\left[g(R) \rho(R)+\frac{M_{\mathrm{T}}(R)}{4 \pi R^{2}} \frac{\mathrm{d} g(R)}{\mathrm{d} R}\right] .
$$

Factorising $g(R) \rho(R)$, we find

$$
n^{2} \Lambda(T)=-\left(\frac{15}{4}\right)\left(\frac{\Omega}{4 \pi}\right) \sigma g(R) \rho(R)\left[1+\frac{\mathrm{d} \ln g(R)}{\mathrm{d} \ln M_{\mathrm{T}}(R)}\right]
$$

The gas distribution in clusters is usually approximated by a $\beta$-profile, which is written

$$
\rho(R)=\frac{\rho_{0}}{\left[1+\left(R / r_{0}\right)^{2}\right]^{\beta}},
$$

where $\rho_{0}$ is the central density and $r_{0}$ is the scale height. Assuming the the gas temperature is independent of radius, then $\nabla p=\left(k_{\mathrm{b}} T / \mu m_{\mathrm{p}}\right) \mathrm{d} \rho / \mathrm{d} R$, so the gravitational accerlation is

$$
g(R)=\frac{\nabla p(R)}{\rho(R)}=-\frac{k_{\mathrm{b}} T}{\mu m_{\mathrm{p}}} \frac{2 \beta R}{\left(r_{0}^{2}+R^{2}\right)},
$$

and equation (34) can be rearranged to obtain the critical cooling time

$$
t_{\mathrm{cool}, \mathrm{crit}} \equiv \frac{5}{2} \frac{n k_{\mathrm{b}} T}{n^{2} \Lambda(T)} \sim\left(\frac{4 \pi}{\Omega}\right)\left(\frac{1}{3 \beta \sigma}\right)\left(\frac{r_{0}^{2}+R^{2}}{R}\right) .
$$


Table 1. Table showing the data used to plot figure 2. Column 1 lists the names of the clusters, column 2 contains the observed X-ray luminosities within the cooling radius, $L_{\mathrm{X}}\left(<r_{\text {cool }}\right)$, column 3 gives the $M_{\mathrm{T}}\left(r_{\text {cool }}\right) g\left(r_{\text {cool }}\right)$ values, derived from temperature and density fits to the data. Column 4 lists the velocity dispersions defined as $\sigma^{2}=k_{\mathrm{b}} T / \mu m_{\mathrm{p}}$.

\begin{tabular}{lccc}
\hline Name & $L_{\mathrm{X}}\left(<r_{\text {cool }}\right) / 10^{42} \mathrm{erg} \mathrm{s}^{-1}$ & $-M_{\mathrm{T}}\left(r_{\text {cool }}\right) g\left(r_{\text {cool }}\right) / 10^{37} \mathrm{~g} \mathrm{~cm} \mathrm{~s}^{-2}$ & $\sigma / \mathrm{km} \mathrm{s}^{-1}$ \\
\hline Virgo & 9.8 & 0.55 & 620 \\
Perseus & 670 & 20 & 1100 \\
Hydra & 250 & 11 & 780 \\
A2597 & 430 & 18 & 800 \\
A2199 & 150 & 3.7 & 830 \\
A1795 & 490 & 23 & 970 \\
A478 & 1220 & 62 & 1100 \\
\hline
\end{tabular}

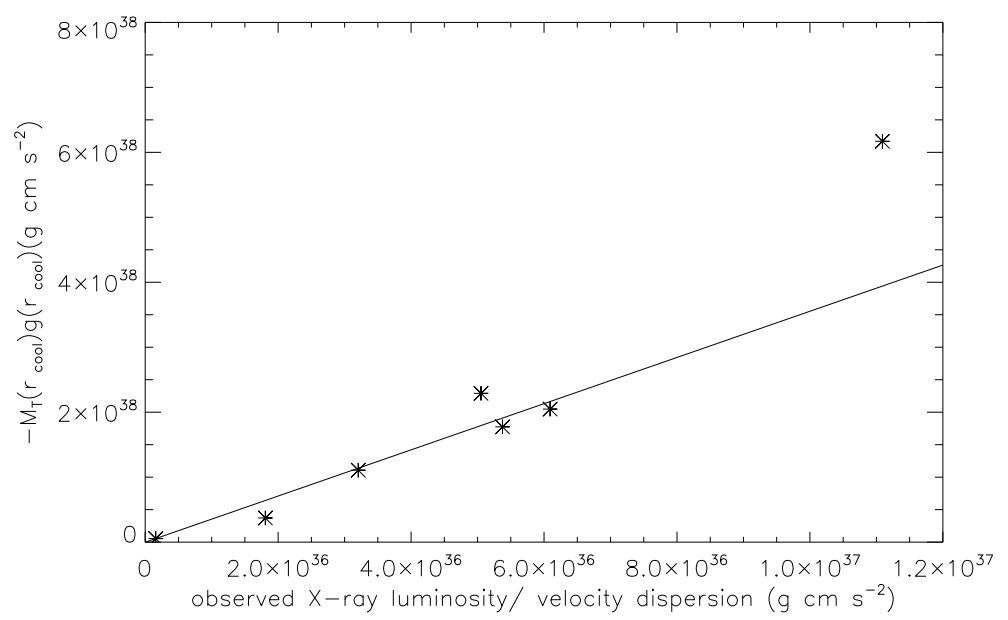

Figure 2. Plot for equation (29) using data for the Virgo, Perseus, Hydra, A2199, A2597, A2199, A1795, A478 taken from Pope, et al. (2006) and references therein. The plot confirms that $M_{\mathrm{T}}\left(r_{\text {cool }}\right) g\left(r_{\text {cool }}\right)$ is linearly related to the X-ray luminosity within the cooling, as the model predicts. The solid line shows the best-fit to the data, which yields an opening angle for the outflow of $\Omega / 4 \pi \sim 0.028$.

The minimum occurs at $R=r_{0}$, at which point the function simplifies to

$$
t_{\mathrm{cool}, \mathrm{crit}} \sim\left(\frac{4 \pi}{\Omega}\right)\left(\frac{1}{3 \beta}\right)\left(\frac{r_{0}}{\sigma}\right) .
$$

This relation is only approximate since it was assumed that the term in square brackets in equation (34) is $\sim 1$. However, the approximation is reasonable because we expect $\mathrm{d} \ln g / \mathrm{d} \ln M_{\mathrm{T}} \sim 0$ near $r_{0}$.

For cooling times below this threshold, material can build up in the core of a cluster, because the energy injection rate is insufficient to drive it away. This build up of material is associated with the star formation observed in clusters with short central cooling times.

As a general example, let $r_{0}=30 \mathrm{kpc}, \sigma=10^{3} \mathrm{~km} \mathrm{~s}^{-1}$, $\beta=3 / 4$ and $\Omega / 4 \pi=0.028$, so that $t_{\text {cool.crit }} \sim 0.5$ Gyr. This agrees well with the value found by Rafferty et al. (2008) below which star formation appears to 'switch on'. Note that this is also similar to the entropy threshold found by Cavagnolo et al. (2008).

In table 2, we list the critical cooling times calculated for the Virgo, Perseus and Hydra clusters. These objects were chosen because $\beta$-profiles are used to fit the gas density profiles, and the clusters are sufficiently resolved that the fits are reliable to small radii. $r_{0}$ and $\beta$ are taken from
Pope et al. (2006), and references therein (see David et al. 2001; Ghizzardi et al. 2004; Sanders et al. 2004). The Virgo and Perseus clusters can be described using a double $\beta$ profile (see Churazov et al. 2003; Ghizzardi et al. 2004) with different scale heights and $\beta$ s. For the Virgo cluster, we took the parameters for the profile with the larger scale height, and used a single $\beta$-profile fit for the Perseus cluster.

Simple analysis also suggests that the ratio $r_{0} / \sigma$ should be fairly constant. According to the virial relations we expect $\sigma^{2} \propto M / r_{0} \propto r_{0}^{2}$, so that $r_{0} / \sigma$ should be approximately constant. As a result, a cooling time of 0.5 Gyr should mark a transition in behaviour in all clusters, as appears to be the case. Whenever, the cooling time of the gas, drops below this critical value, we should expect to observe the consequences of material building up in the centre of a cluster.

It has been suggested by Cavagnolo et al. (2008) and Soker (2008) that the presence of the critical cooling time is a possible indication that central AGN are fuelled by the cold feedback mechanism (Pizzolato \& Soker 2005). Instead, the model presented here suggests that the phenomenon occurs directly because of the buoyancy injected by the AGN. This places no requirements on the AGN fuelling mechanism, except that it must balance the radiative losses within 
Table 2. Table showing the data for the critical cooling time. Column 1 gives the cluster name, column 2 lists the core radius of the density profile, column 3 gives the $\beta$ value of the density profile and column 4 shows the critical cooling time according to equation (37), using the appropriate $\sigma$ values from table 1 . The opening angle was taken to be $\Omega / 4 \pi=0.028$, from the best-fit to equation (29). The values of $r_{0}$ and $\beta$ are taken from Pope, et al.(2006), and references therein.

\begin{tabular}{lccc}
\hline Name & $r_{0} / \mathrm{kpc}$ & $\beta$ & $t_{\text {cool }, \text { crit }} / 10^{9} \mathrm{yrs}$ \\
\hline Virgo & 23.3 & 0.71 & 0.5 \\
Perseus & 28.5 & 0.81 & 0.3 \\
Hydra & 18.6 & 0.72 & 0.4 \\
\hline
\end{tabular}

the cooling radius. Cold feedback still provides a possible mechanism for achieving this.

An alternative view, presented by Voit et al. (2008), is that the onset of star formation and optical emission occur below a critical entropy. They showed that for a central entropy greater than $K_{0} \sim 30 \mathrm{keVcm}^{2}$, thermal conduction transports energy at a rate greater than can be radiated away by the hot gas, assuming that the conduction is not heavily suppressed. In this case entropy is defined as $K=k_{\mathrm{b}} T n_{\mathrm{e}}^{-2 / 3}$ (e.g. Lloyd-Davies et al. 2000), where $k_{\mathrm{b}}$ is the Boltzmann constant, $T$ is the temperature and $n_{\mathrm{e}}$ is the electron number density. Material below the critical entropy would be thermally unstable, able to cool, and could potentially fuel the central AGN. This explanation fits neatly into the framework outlined by Guo et al. (2008) in which non cool-core clusters can be stabilised by thermal conduction, whereas cool core clusters require AGN feedback and thermal conduction for stability. Their view is not inconsistent with the model presented here. For example, the model discussed here focusses on clusters in which AGN feedback is important, and thermal conduction cannot balance radiative cooling. However, complete consistency would require that AGN heating either i) does not raise the central entropy above $\sim 30 \mathrm{keVcm}^{2}$, otherwise the gas would never be able to cool to fuel the AGN again, or ii) that thermal conduction is heavily suppressed.

One difficulty with the possibility suggested by Voit et al. (2008) is that the value of the thermal conduction suppression factor is highly uncertain. In contrast, the model presented here predicts a critical cooling time which contains no free parameters. Therefore, probably the best way to distinguish between the models would be to determine whether, and by how much, thermal conduction is suppressed. Unfortunately, this is a question that cannot easily be answered.

If the model presented here does describe some of the real mechanisms at work in clusters, perhaps the most important aspect to highlight is the self-tuning ability of AGN feedback. This is true in both elliptical galaxies and clusters. The tuning is achieved by slightly different mechanisms in each case. In elliptical galaxies, an equilibrium is reached when the AGN has ejected so much gas that its fuel supply becomes limited and it can no longer exceed the critical threshold required to eject gas from the gravitational potential. In clusters, the AGN injects energy at a particular rate given by the details of the accretion process. This energy injection has the twin effect of heating the gas and also moving it about within the cluster potential. Within the radius that the AGN heating balances the radiative losses, the heating also controls the weight of cooling material. and therefore its own fuel supply.

\section{SUMMARY}

We have constructed an analytical model to investigate the observable effects of kinetic and thermal AGN feedback on elliptical galaxies and galaxy clusters. The results show that there is a critical momentum injection rate above which an AGN outflow will eject mass from an elliptical galaxy. For an AGN fuelled at a rate proportional to the classical mass cooling rate, this leads to $L_{\mathrm{X}} \propto \sigma^{10}$ which agrees well with observations (e.g. Mahdavi \& Geller 2001) and reasonably with simulations (e.g. Bower et al. 2008; Davé et al. 2008). If the AGN fuelling rate is only proportional to $L_{\mathrm{X}}$ this would lead to $L_{\mathrm{X}} \propto \sigma^{8}$. Presumeably these represent the extremes, so that a different fuelling mechanism might lead to an intermediate scaling.

In more massive environments, the work done by thermal energy injected by the AGN also becomes significant. This mechanism should lead to $L_{\mathrm{X}} \propto \sigma^{7}$, assuming the AGN fuelling rate is proportional to the classical mass cooling rate. It is not clear whether the observations confirm this, due to the large scatter. Again, if the AGN fuelling rate is $\propto L_{\mathrm{X}}$, this would lead to $L_{\mathrm{X}} \propto \sigma^{5}$. Scalings between $\sigma^{5}$ and $\sigma^{7}$ are also, no doubt, possible in this regime, depending on the details of the AGN fuelling mechanism.

Above $\sigma \sim 500 \mathrm{~km} \mathrm{~s}^{-1}$ AGN heating of either sort is unable to affect the global $L_{\mathrm{X}}-\sigma$ relation. This corresponds to a temperature of roughly $1-2 \mathrm{keV}$ and can explain the observed break in the $L_{\mathrm{X}}-T$ relation (e.g. Xue \& Wu 2000). In order for this break to be noticeable, it is required that the $L_{\mathrm{X}}-\sigma$ relation is steeper for ellipticals than for clusters. Since $L_{\mathrm{X}} \propto \sigma^{4.4}$ in clusters (Mahdavi \& Geller 2001), this implies that the AGN fuelling rate probably does scales more like $L_{\mathrm{X}} / \sigma^{2}$ than $L_{\mathrm{X}}$.

In clusters of galaxies, the stronger gravity means that outflows become buoyancy dominated above a certain length scale. Simple estimates suggest that $\sim 20 \mathrm{kpc}$ might be typical. It has also be shown that buoyancy is equivalent to the work done by thermal energy injection and since typical cooling radii in clusters are $\sim 100 \mathrm{kpc}$, global considerations of AGN heating in clusters can be treated as if the energy injection is purely thermal.

It is not possible for an AGN to completely eject material from a cluster, however, it is possible for the energy injection to redistribute material within the central regions of the cluster. This can lead to the self-tuning of AGN feedback so that heating roughly matches cooling within the cooling 
radius. A direct consequence of this is that the mass of hot material within the cooling radius should be proportional to $L_{\mathrm{X}}\left(<r_{\text {cool }}\right) /\left[\sigma g\left(r_{\text {cool }}\right)\right]$. This appears to be true, at least for a sample of seven nearby clusters. The only possible exception is A478, which also displays evidence of a strong inflow and minimal AGN heating and might therefore be expected to contain excess material in the central regions. Furthermore, the same physical processes lead to the derivation of a critical cooling time of $\sim 0.5 \mathrm{Gyr}$, below which material can pile up in the cluster centre. This is very close to the observationally determined universal cooling time of $\sim 0.5$ Gyr, found by Rafferty et al. (2008), below which star formation and AGN activity appear to be triggered.

\section{ACKNOWLEDGEMENTS}

I would like to thank the Department of Foreign Affairs and International Trade for funding through a Government of Canada Post-Doctoral Research Fellowship and also CITA for funding through a National Fellowship. I also thank Arif Babul for informative discussions and additional funding, and the anonymous referee for positive, helpful comments that improved this work.

\section{REFERENCES}

Bîrzan L., Rafferty D. A., McNamara B. R., Wise M. W., Nulsen P. E. J., 2004, ApJ, 607, 800

Benson A. J., Bower R. G., Frenk C. S., Lacey C. G., Baugh C. M., Cole S., 2003, ApJ, 599, 38

Best P. N., 2007, New Astronomy Review, 51, 168

Best P. N., Kauffmann G., Heckman T. M., Brinchmann J., Charlot S., Ivezić Ž., White S. D. M., 2005, MNRAS, 362,25

Bicknell G. V., 1994, ApJ, 422, 542

Binney J., Bibi F. A., Omma H., 2007, MNRAS, 377, 142

Binney J., Tabor G., 1995, MNRAS, 276, 663

Bower R. G., Benson A. J., Malbon R., Helly J. C., Frenk C. S., Baugh C. M., Cole S., Lacey C. G., 2006, MNRAS, 370,645

Bower R. G., McCarthy I. G., Benson A. J., 2008, MNRAS, 390, 1399

Brüggen M., 2003, ApJ, 593, 700

Bregman J. N., David L. P., 1988, ApJ, 326, 639

Burns J. O., 1990, ApJ, 99, 14

Carlotti P., Hunt G. R., 2005, Journal of Fluid Mechanics, 538,343

Cavagnolo K. W., Donahue M., Voit G. M., Sun M., 2008, ApJ, 683, L107

Churazov E., Forman W., Jones C., Böhringer H., 2003, ApJ, 590, 225

Crawford C. S., Allen S. W., Ebeling H., Edge A. C., Fabian A. C., 1999, MNRAS, 306, 857

Croton D. J., Springel V., White S. D. M., De Lucia G., Frenk C. S., Gao L., Jenkins A., Kauffmann G., Navarro J. F., Yoshida N., 2006, MNRAS, 365, 11

Davé R., Oppenheimer B. D., Sivanandam S., 2008, MNRAS, 391, 110
David L. P., Nulsen P. E. J., McNamara B. R., Forman W., Jones C., Ponman T., Robertson B., Wise M., 2001, ApJ, 557,546

De Young D. S., 1993, ApJ, 405, L13

Diehl S., Li H., Fryer C. L., Rafferty D., 2008, ApJ, 687, 173

Diehl S., Statler T. S., 2008, ApJ, 680, 897

Dunn R. J. H., Fabian A. C., Taylor G. B., 2005, MNRAS, 364,1343

Fukazawa Y., Botoya-Nonesa J. G., Pu J., Ohto A., Kawano N., 2006, ApJ, 636, 698

Gaetz T. J., 1989, ApJ, 345, 666

Ghizzardi S., Molendi S., Pizzolato F., De Grandi S., 2004, ApJ, 609, 638

Guo F., Oh S. P., Ruszkowski M., 2008, ApJ, 688, 859

Heinz S., Brüggen M., Young A., Levesque E., 2006, MNRAS, 373, L65

Hoeft M., Brüggen M., 2004, ApJ, 617, 896

Kaiser C. R., Best P. N., 2007, MNRAS, 381, 1548

King A., 2003, ApJ, 596, L27

King A., 2005, ApJ, 635, L121

Laing R. A., Bridle A. H., 2002, MNRAS, 336, 1161

Lloyd-Davies E. J., Ponman T. J., Cannon D. B., 2000, MNRAS, 315, 689

Maccarone T. J., Gallo E., Fender R., 2003, MNRAS, 345, L19

Mahdavi A., Geller M. J., 2001, ApJ, 554, L129

McLure R. J., Willott C. J., Jarvis M. J., Rawlings S., Hill G. J., Mitchell E., Dunlop J. S., Wold M., 2004, MNRAS, 351,347

Meier D. L., 1999, ApJ, 522, 753

Mittal R., Hudson D. S., Reiprich T. H., Clarke T., 2008, ArXiv e-prints

Murray N., Quataert E., Thompson T. A., 2005, ApJ, 618, 569

Nulsen P. E. J., Jones C., Forman W. R., David L. P., McNamara B. R., Rafferty D. A., Bîrzan L., Wise M. W., 2007, in Böhringer H., Pratt G. W., Finoguenov A., Schuecker P., eds, Heating versus Cooling in Galaxies and Clusters of Galaxies AGN Heating Through Cavities and Shocks. pp 210-+

Nusser A., Silk J., Babul A., 2006, MNRAS, 373, 739

O'Sullivan E., Forbes D. A., Ponman T. J., 2001, MNRAS, 328,461

O'Sullivan E., Ponman T. J., Collins R. S., 2003, MNRAS, 340,1375

Pavlovski G., Kaiser C. R., Pope E. C. D., 2007, ArXiv e-prints

Pavlovski G., Kaiser C. R., Pope E. C. D., Fangohr H., 2008, MNRAS, 384, 1377

Pizzolato F., Soker N., 2005, ApJ, 632, 821

Pope E. C. D., Pavlovski G., Kaiser C. R., Fangohr H., 2005, MNRAS, 364, 13

Pope E. C. D., Pavlovski G., Kaiser C. R., Fangohr H., 2006, MNRAS, 367, 1121

Puchwein E., Sijacki D., Springel V., 2008, ApJ, 687, L53

Rafferty D. A., McNamara B. R., Nulsen P. E. J., 2008, ApJ, 687, 899

Rafferty D. A., McNamara B. R., Nulsen P. E. J., Wise M. W., 2006, ApJ, 652, 216

Roychowdhury S., Ruszkowski M., Nath B. B., Begelman M. C., 2004, ApJ, 615, 681 
Ruszkowski M., Begelman M. C., 2002, ApJ, 581, 223

Sanders J. S., Fabian A. C., Allen S. W., Schmidt R. W., 2004, MNRAS, 349, 952

Soker N., 2008, ApJ, 684, L5

Spergel D. N., Bean R., Doré O., Nolta M. R., Bennett C. L., Dunkley J., Hinshaw G., Jarosik N., Komatsu E., Page L., Peiris H. V., Verde L., Halpern M., Hill R. S., Kogut A., 2007, ApJ Supp., 170, 377

Sun M., Jones C., Murray S. S., Allen S. W., Fabian A. C., Edge A. C., 2003, ApJ, 587, 619

Tabor G., Binney J., 1993, MNRAS, 263, 323

Turner J. S., 1986, Journal of Fluid Mechanics, 173, 431

Voigt L. M., Fabian A. C., 2004, MNRAS, 347, 1130

Voit G. M., Cavagnolo K. W., Donahue M., Rafferty D. A., McNamara B. R., Nulsen P. E. J., 2008, ApJ, 681, L5

Voit G. M., Donahue M., 2005, ApJ, 634, 955

Xue Y.-J., Wu X.-P., 2000, ApJ, 538, 65 